\title{
Numerical Simulating of the Interaction of Vertical Fixed Multiple Membranes with Gravity Wave
}

\author{
Jia-Wei $\mathrm{TAO}^{1, \text { a }}$, Jing-Ping $\mathrm{WU}^{2, \mathrm{~b}^{*}}$, Tian-Long $\mathrm{MEI}^{3, \mathrm{c}}$, Ling $\mathrm{XU}^{4, \mathrm{~d}}$ \\ 1,2,3,4 School of Transportation, Wuhan University of Technology, Wuhan, China \\ axiaotaodaren@sina.cn, bwujp@whut.edu.cn, ctackimay@163.com, dlanshing90@163.com \\ ${ }^{*}$ Corresponding author
} Keywords: Wave Attenuation, Bragg Resonace, Membrances, Numerical Simulation, Floating
Breakwater.

\begin{abstract}
In deep sea or temporary application, the floating breakwater should be best used to attenuate the surface wave. But the most difficulty of floating breakwater is to decrease long wave length wave. Bragg resonance condition appears while incident surface water wavelengths are approximately twice the periodic structure wavelengths, and large amounts of incident wave energy are reflected. In recent years, flexible membrane structure, with its numerous advantages, has attracted the attention of the coastal and ocean engineers and researchers. Although Bragg resonance is investigated by using diffirent analytical methods, it is not found by using CFD method. Therefore, in this paper, we use commercial software code FLUENT to numerical simulating the interaction of surface grivaty wave with multiple veitically fixed membranes with constant gaps. At the beginning, we verified the numerical technology by simulating the wave making and the interaction of wave with single membrane; then, we computed 4 diffirent length veitically fixed multiple membranes. The results show that the transmission coefficient is small and decline obviously with the membrane lengthened when incident surface water wavelengths are approximately twice the membrane gap. However, we need to simulate more wave lengthes to find whether there is the strong reflection induced by Bragg resonance. The works in this paper are the base about more studies on Bragg resonance being applied to floating breakwater.
\end{abstract}

\section{Introduction}

In deep sea or temporary application, the floating breakwater should be best used to attenuate the surface wave. But the most difficulty of floating breakwater is to decrease long wave length wave ${ }^{[1]}$.

Bragg resonance was first discovered in crystallography[2].

In coastal and ocean engineering, Heathershaw[3] first published laboratory measurements that significant and large amounts of wave energy be reflected by resonant interactions between surface-water waves and the undulating bedforms while incident surface water wavelengths are approximately twice the bedform wavelengths; Mei[4] explain a theory that strong reflection can be induced by the sandbars themselves, once the so-called Bragg resonance condition is met. Chou[5] extended Floquet's Theorem to derive dispersion relations for small-amplitude water wave propagation in the presence of an infinite array of periodically arranged surface scatterers. For one-dimensional periodicity(stripes), band gaps are found for wavevectors in the direction of periodicity corresponding to frequency ranges which support only non-propagating standing waves, as a consequence of multiple Bragg scattering. Bennetts[6] constructed a three-dimensional model of ocean-wave scattering in the marginal ice zone by using linear theory under time-harmonic conditions. Bragg resonance is identified when the rows are identical and equi-spaced. Karmakar[7] analyzed the interaction of surface gravity waves with multiple veitically moored surface-piercing membrane breakwaters in finite water depth based on the linearized theory of water waves. The resonating pattern in the reflection coefficients is observed for multiple floating membrane which can be referred as Bragg's resonance.

In recent decades, flexible floating membrane breakwaters are preferred popularly as they are easy to carry, inexpensive, reusable, rapidly deployable and removable, and less environmental 
impacts[7][8]. Although Bragg resonance is investigated by using diffirent analytical methods, it is not simulated-by using CFD method. Therefore, in this paper, we use commercial software code FLUENT to numerical simulating the interaction of surface grivaty wave with veitically fixed multiple membranes with constant gaps, however, the so-called membrane only stand for thin with no deflection. At the beginning, we verified the numerical technology by simulating the wave making and the interaction between wave and single membrane, see 4.1 ; next, we computed 4 diffirent length multiple veitically fixed membranes, see 4.2. While incident surface water wavelengths are approximately twice the membrane gap, the transmission coefficient is small and decline obviously with the membrane lengthened. However, we need to compute more wave lengthes to claim whether the interactions between the mulitiple membranes with $1 \mathrm{~m}$ interval gaps and $\lambda=1.8 \mathrm{~m}, 2 \mathrm{~m}, 2.2 \mathrm{~m}$ waves are induced by Bragg resonance. The works in this paper are the base about more studies on Bragg resonance being applied to floating breakwater.

\section{Governing Equations and Numerical Method}

Assuming fluid is incompression, the governing equations include the continuity equation and N-S equations[9]:

$$
\begin{aligned}
& \nabla \vec{v}=0, \\
& \frac{\partial}{\partial t}(\rho \vec{v})+\nabla(\rho \vec{v} \vec{v})=\rho \vec{g}-\nabla p+\mu \nabla^{2} \vec{v}+\vec{F},
\end{aligned}
$$

Where $\rho$ is the fluid density, and $\vec{v}$ is the velocity vector, $g$ is the acceleration of gravity, $p$ is the static pressure, $\mu$ is the viscosity, $\vec{F}$ is external forces.

The VOF (Volume of Fluid) technique is used to determine the location of the interface between air and water ${ }^{[10]}$.

$$
\frac{\partial \alpha_{q}}{\partial t}+\nabla\left(\alpha_{q} \vec{v}\right)=0
$$

Where $\alpha_{q}$ is the volume fraction function of the cell in the computing domain, $q=1,2$ indicates the fluid phase, water or air.

FLUENT solver is based on the finite volume method. For a free surface problem simulating, the free surface elevation is captured by Geometric Reconstruction technology, the Pressure-Velocity Coupling scheme uses PISO algorithm, Pressure Discretization uses PRESTO! Scheme, and the convergence criterion set at $10^{-6}$. In this paper, the fluid viscosity is ignored in fact to avoid wall boundary mesh and improve computing speed.

\section{Numerical Model}

\section{Numerical Wave Tank and Boundary Conditions}

The water depth $\mathrm{h}$ of the numerical wave tank is same as the depth of the physical wave flume in our laboratory, $h=0.5 \mathrm{~m}$; the tank length $\mathrm{L}$ is based on the wave reflection form the membranes, the inlet and outlet boundary, $L \geq 15 \lambda, \lambda$ is the wave length; the tank height is $1 \mathrm{~m}$; there is a two wave lengths fragment wave beach at the tank end.

In Fig. 1, the numerical wave tank is shown schematically.

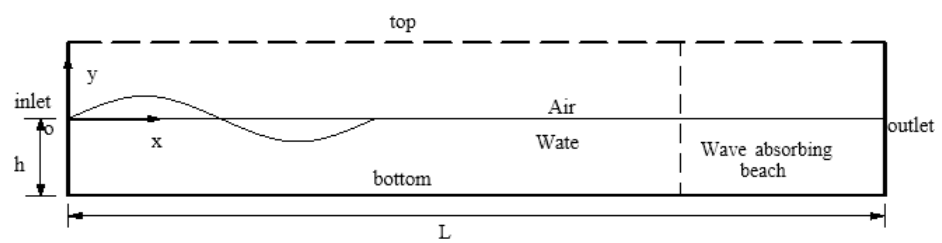

Fig. 1 Schematic Representation of the Wave Tank 
The wave tank consisits of four boundarys: inlet, outlet, top and bottom. The inlet is defined as velocity inlet, both the outlet and the top as pressure outlet, and the bottom as symmetry condition practically.

The inlet is the wave making boundary, the gauge pressure of top is zero, the gauge pressure of the outlet is linear hydrostatic pressure distribution.

\section{Computational Model and Mesh}

Firstly, the wave making technology of the numerical wave tank has been verified. Fig. 2 shows the model and mesh for making a wave of $\lambda=2 m$ wave, tank length $L$ is $30 \mathrm{~m}$,water depth $h$ is $0.5 \mathrm{~m}$.

Then, in order to test the numerical technology for the simulation of wave and membrane interaction, we compared the transmission coefficient of the interaction between single membrane and surface gravity wave to Karmakar[7]'s analytical solution.Fig. 3 shows this model and mesh, the tank scale is as same as Fig. 2, and the membrane is fixed at $5 \lambda$ far away from the inlet, its length is $l=25 \mathrm{~cm}$ from free surface downward.

At last, based on Bragg resonance and Heathershaw's $10 \times 1 \mathrm{~m}$ wavelength and $5 \mathrm{~cm}$ amplitude sinusoidal bars model[3], we studied 10 membranes with 4 different length and $1 \mathrm{~m}$ constant gap between two neighboring membranes. Three length of the four are $l=5,10,15 \mathrm{~cm}$ respectively from free surface downward; the other one is $l=6.25 \mathrm{~cm}, 1.25 \mathrm{~cm}$ from free suface upward and $5 \mathrm{~cm}$ from free surface downward. The multiple membranes are fixed and the membranes are no deflection. Fig. 4 shows $l=15 \mathrm{~cm}$ the one model of the four for $\lambda=2 \mathrm{~m}$ wave, $L=39 \mathrm{~m}, h=0.5 \mathrm{~m}$.

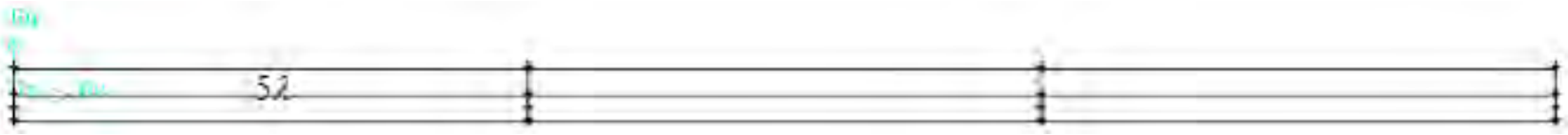

Fig. 2 Wave Tank Model without Membrane

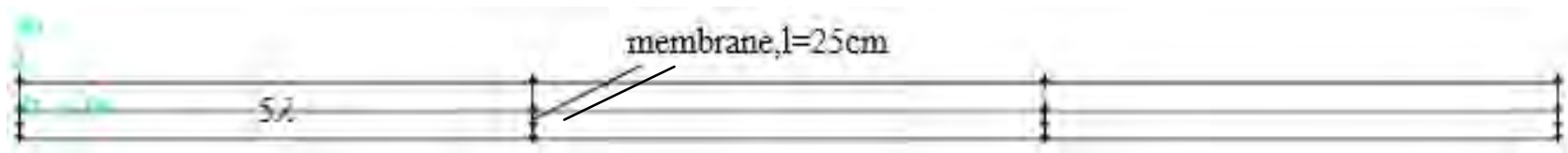

Fig. 3 Wave Tank Model with Single Membrane, $l=25 \mathrm{~cm}$

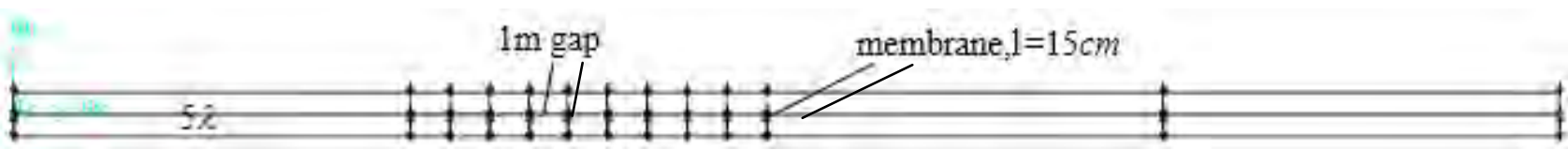

Fig. 4 Wave Tank Model with 10 Multiple Membranes, $l=15 \mathrm{~cm}$

The mesh generation follows that $\Delta y=A / 20$ at the area of $y= \pm 1.5 A$, A is wave amplitude, $\Delta x=\lambda / 80$, and $\Delta x$ increase while near the tank outlet.

\section{Result and Discussion}

\section{Numerical Simulation Verification}

Although Gomes[9] and $\mathrm{Du}[10]$ used commercial software code FLUENT to generate regular waves achived to the desired characteristics, we still need to do the verifications for the numerical simulations of both wave making and the interaction between gravity wave and membrane.

Wave Making.In order to grasp the accuracy extent of both time-space varied wave contour and the single membrance transmission coefficient, $\lambda=0.628,1,1.5,2,2.5,4,6.7 m$ with constant wave steep 0.005 are computed. And $\lambda=2 m$ wave is taken for a example in the following figures.

The theoretical expression of wave elevation $\eta$ is a Second-order Stokes wave: 


$$
\eta=A \cos (k x-\sigma)+\frac{A^{2} k}{4} \frac{\cosh k h}{\sinh ^{3} k h}(2+\cosh 2 k h) \cos 2(k x-\sigma t),
$$

$A$ is wave amplitude, $k$ is wave number, $\sigma$ is circular frequency.

Fig. 5 gives the wave elevation history $\eta / A$ at $x=\lambda$ from the time of 0 to $t / T=20.291, T$ is wave period. The numerical result matches the theoretical result well.

Fig. 6 and Fig. 7 give the water velocity distribution along water depth at $x=0$ and $x=\lambda$ respectively under some instant. Regard for the velocity of y direction, the present numerical results agree with the theory results very well. However, for the velocity of $\mathrm{x}$ direction, although below the free surface, the present numerical results have a satisfied agreement with the theory results, near the free suface, the present numerical results appear rapid recrease. We are planning to apply PIV technology to measure the $\mathrm{x}$ velocity near the free surface.

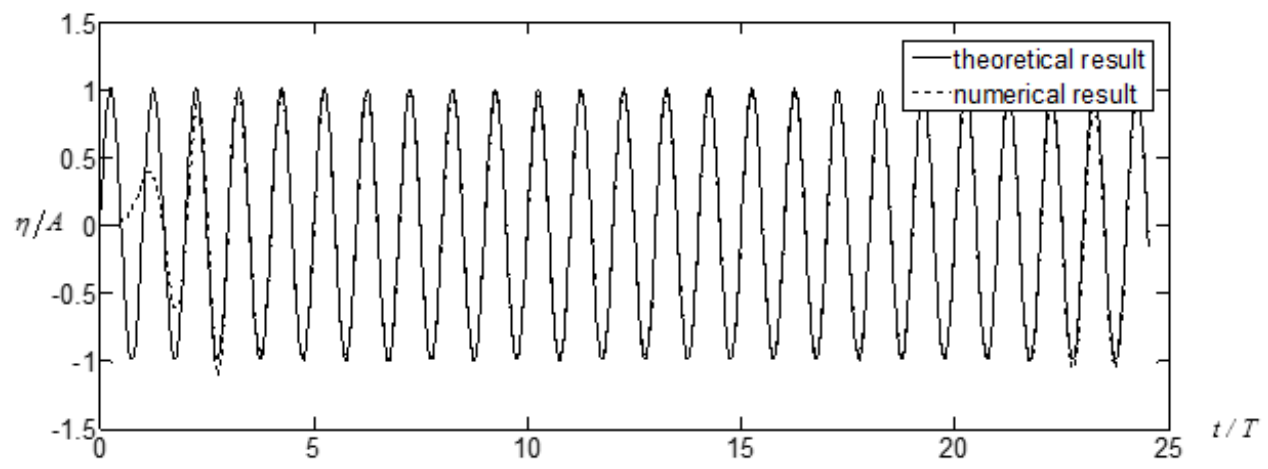

Fig. 5 Wave Elevation History $\eta / A$ at $x=\lambda, \lambda=2 m$

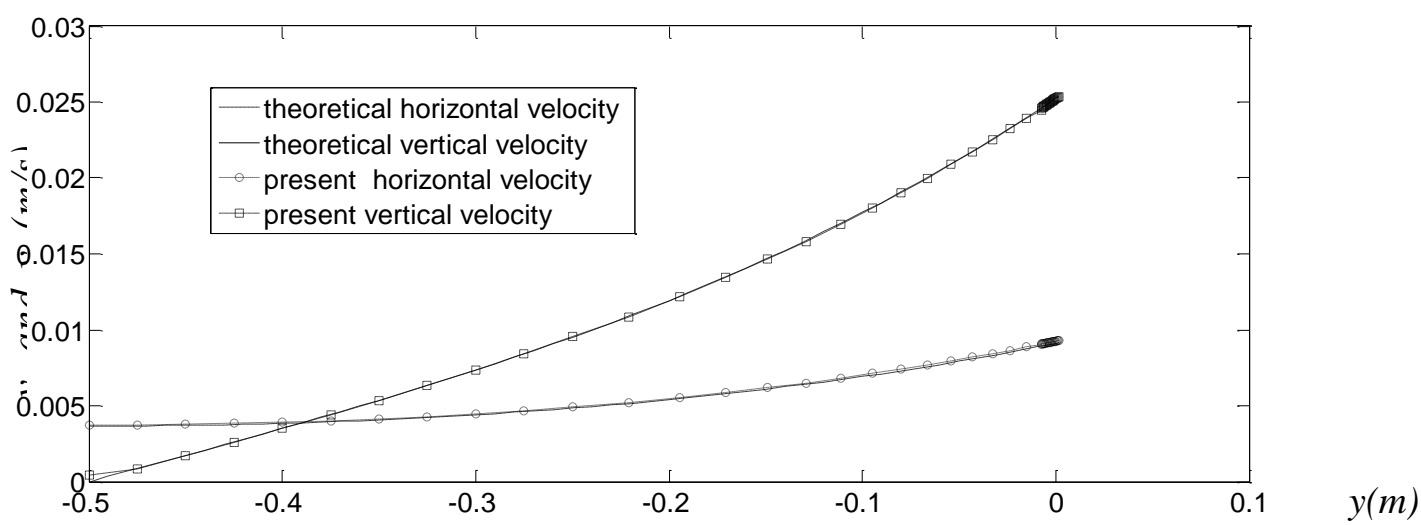

Fig. 6 Horizontal Velocity vx and Vertical Velocity vy at $x=0, \lambda=2 m$

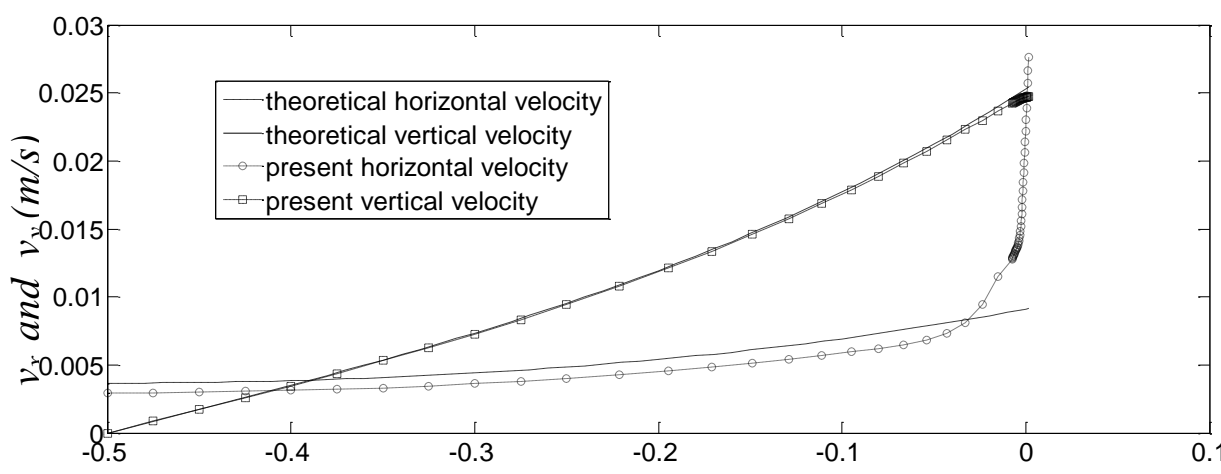

$y(m)$

Fig. 7 Horizontal Velocity vx and Vertical Velocity vy at $x=\lambda, \lambda=2 m$

Transmission Coefficient of Single Membrane.The single membrane in this paper is simplified from Karmakar. Our membrane is $25 \mathrm{~cm}$ length and fixed from free surface downward with no 
deflection. Karmakar's membrane is $l / h=0.5$, and the membrane is porous and takes deflection with fixed edge at $y=0$ and moored edge at $y=-l$.

The interaction of single membrane with $\lambda=0.628,1,1.5,2,2.5,4,6.7 \mathrm{~m}$ waves are simulated, and the transmission coefficients are dealt out. Fig. 8 gives the numerical results compared with Karmakar[7]'s analytical solution. The numerical results have a satisfied agreement with Karmakar's result although.

The transmission coefficient $K_{t}$ is: $K_{t}=\frac{A_{t}}{A}, A_{t}$ is the transmitted wave amplitude.

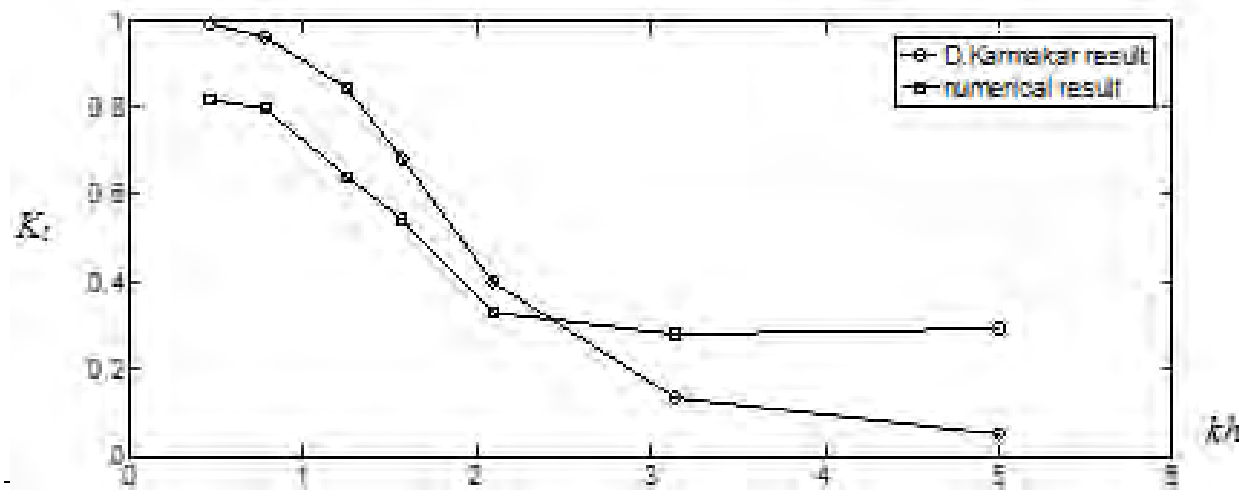

Fig. 8 The Transmission Coefficients of Single Membrane

\section{Transmission Coefficient of Multiple Membranes}

The parameter of our multiple membranes is from Heathershaw's bars model. Heathershaw ${ }^{[3]}$ 's bars model was a $10-\mathrm{m}$ long test section consisting of $10 \times 1 \mathrm{~m}$ wavelength, $0.05 \mathrm{~m}$ amplitude sinusoidal bars and set in a false bottom.

At first, we constructed 10 membranes of $l=5 \mathrm{~cm}$ from free surface downward with $1 \mathrm{~m}$ constant gap between two neighboring membranes. But the transmission coefficients show that wave reflection is very litlle,even at $\lambda=2 m$ which Bragg resonance should be taken place, see Fig. 9.

Then we tried two directions to add 10 membranes length: upward and downward. The one was added $1.25 \mathrm{~cm}$ upward, $l=6.25 \mathrm{~cm}$; and the other one was added $5 \mathrm{~cm}$ downward, $l=10 \mathrm{~cm}$. Because of the expense of computing time, only $\lambda=1.8 \mathrm{~m}, 2 \mathrm{~m}, 2.2 \mathrm{~m}$ are choosed for simulating.

From Fig. 9, the transmission coefficients of $l=6.25 \mathrm{~cm}$ have almost no changes with $l=5 \mathrm{~cm}$, it gives that the length above free surface do little effect here; on the other hand, the transmission coefficients of $l=10 \mathrm{~cm}$ fall obviously. $l=15 \mathrm{~cm}$ are computed at $\lambda=1.8 \mathrm{~m}, 2 \mathrm{~m}, 2.2 \mathrm{~m}$ also.

Fig. 9 shows the transmission coefficients of these 4 models. With the membrane length under free surface increasing, the transmission coefficients decrease apparently. Although the numerical dissipation effect the acuuracy of the transmission coefficients, the decreasing trend is credible.

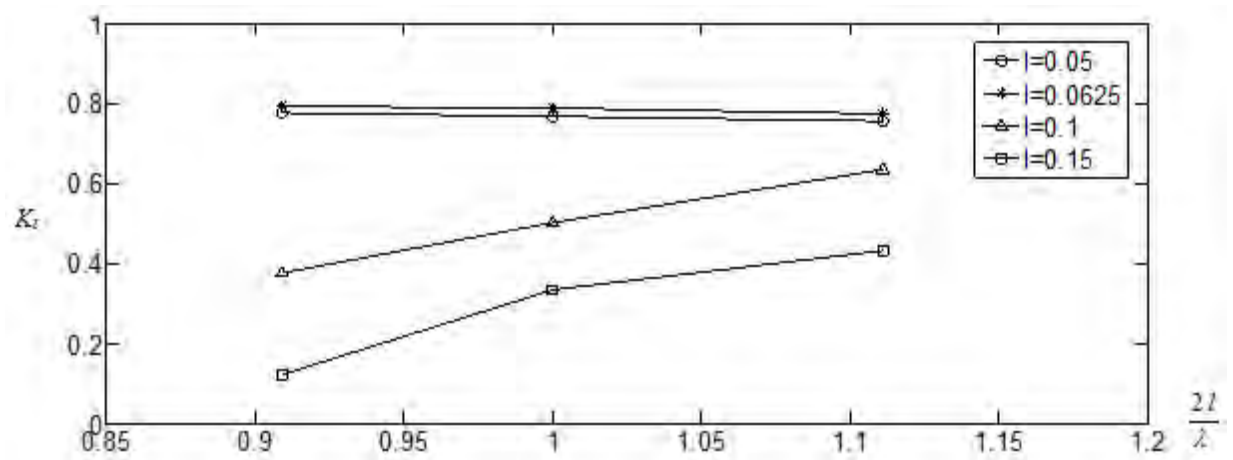

Fig. 9 The Transmission Coefficients of Multiple Membranes

But we need to compute more waves that its wave length is far from $\lambda=2 m$, get the varied trend of the transmission coefficients with the wave length, then we might have the possibility to tell that 
whether it is Bragg resonace's contribution to the smaller transmission coefficients when the the multiple membranes interacting with $\lambda=1.8 m, 2 m, 2.2 m$ waves. And we will give the reflection coefficients in the future.

\section{Conclusion}

In this paper, the commercial software code FLUENT has been applied to numerical simulating the ineraction of gravity wave with membrane. The validation of numerical wave tank results show that the transmission coefficients of wave scattering through single membrane are calculated are found to be in satisfied agreement with Karmakar's analytical solutions. We computed the $1 \mathrm{~m}$-gaps multiple membranes interacting with $\lambda=1.8 m, 2 m, 2.2 m$ waves, the transmission coefficients decrease apparently with the increasing of membrane length under free surface,although there are numerical dissipation. However, we need to simulate more wave lengthes to find the strong reflection factors induced by Bragg resonance.

\section{Acknowledgement}

This work is supported by Natural Science Foundation of Hubei Province of China (No.2012FFB05114).

\section{References}

[1]H.M.TEH, Hydraulic Performance of Free Surface Breakwaters:A Review, Sains Malaysiana, 42(9)(2013), 1301-1310.

[2]Hsien-Kuo Chang, Jin-Cheng Liou, Long wave reflection from submerged trapezoidal breakwaters, Ocean Engineering, 34(2007), 185-191.

[3]A.D.Heathershaw, Seabed-wave resonance and sand bar growth, Nature, 296(1982), 343-345.

Chiang C.Mei, Resonant reflection of surface water waves by periodic sandbars, J.Fluid Mech, 152(1985), 315-335.

[4]Tom Zhou, Band structure of surface flexural-gravity waves along periodic interfaces, J.Fluid Mech, Cambridge University Press, 369(1998), 333-350.

[5]L.G.Bennetts, V.A.Squire, Wave scattering by multiple rows of circular ice floes, J.Fluid Mech, Cambridge University Press, 639(2009), 213-238.

[6]D.Karmakar, J.Bhattacharjee, C Guedes Soares, Scattering of gravity waves by multiple surface-piercing floating membrane, Applied Ocean Research, 39(2012), 40-52.

[7]D.Karmakar, C Guedes Soares, Oblique scattering of gravity waves by moored floating membrane with changes in bottom topography, Ocean Engineering, 54(2012), 87-100.

[8]M.N.Gomes, C.R.Olinto, L.A.O.Rocha, J.A.Souza and L.A.Isoldi, Computational modeling of a regular wave tank, Engenharia Termica, 8(1)(2009), 44-50.

[9]Qingjie.Du, Y.C.Dennis.Leung, 2D numerical simulation of ocean waves, Marine and Ocean Technology, (2011), 2183-2189. 